\title{
Modelling the cyclic response of structural steel for FEM analyses
}

\author{
Ciprian Ionut Zub ${ }^{1, *}$, Aurel Stratan ${ }^{1}$, and Dan Dubina ${ }^{1,2}$ \\ ${ }^{1}$ Politehnica U niversity of Timisoara, D epartment of Steel Structures and Structural M echanics, \\ Timisoara, Romania \\ ${ }^{2}$ Romanian A cademy, Fundamental and A dvanced Technical Research Centre, Timisoara, Romania
}

\begin{abstract}
Modelling the cyclic response of structural steel plays an important role in the design and performance assessment of steel structures. Up to date, several mathematical models were developed to simulate metal plasticity, but only some of them were implemented in Finite Element M ethod (FEM) based software packages such as A baqus, by using incremental plasticity procedures. Within this article, the "builtin" combined isotropic/kinematic hardening model is used to model metal plasticity under cyclic loading regime. A brief description of the constitutive model together with the calibration procedure of the material parameters based on experimental data are presented. Finite element analyses were carried out on simplified FEM models to provide numerical predictions using the calibrated material parameters. Since the "built-in" combined model has several limitations (especially related to the isotropic component), adjustments of the material parameters were made to accommodate to different loading histories. The chosen material model and the calibrated input parameters are validated by analysing the FEM predictions to be in good agreement with the experimental results with respect to cyclic behaviour and failure mode.
\end{abstract}

\section{Introduction}

Modelling the cyclic response of structural steel plays an important role in the design and performance assessment of steel structures. During a seismic event, the structural steel elements (e.g. Buckling Restrained Braces, shear links/panels) are undergoing cyclic deformations. Therefore, a material model able to properly simulate the cyclic behaviour of structural steel must be experimentally validated. The calibrated material model can be further used to simulate the structural elements for cyclic numerical analyses.

The main objective of this article is to provide a modelling technique of the cyclic response of structural steel for FEM analyses using Abaqus "built-in" material models. Specific guidelines are given regarding the calibration of material parameters based on experimental data since the case study is a mild carbon steel with yield plateau (S355).

\footnotetext{
* Corresponding author: ciprian.zub@ student.upt.ro
} 


\section{Cyclic response of steel}

Up to date, several mathematical models were developed to simulate metal plasticity [1]. Only the kinematic hardening models are appropriate to simulate the structural steels under cyclic loading conditions [1] due to the presence of the Bauschinger effect. This effect can be experimentally observed when performing uniaxial cyclic coupon tests under symmetric strain control. As presented in Fig. 1 (a) and (b), the Bauschinger effect consists in decreasing the yield strength, $\sigma^{0}$, when the direction of strain is changed. When simulating structural mild carbon steels, using only the kinematic hardening model is not sufficient to properly simulate the cyclic plastic behaviour, due to several specific aspects.

The first aspect is the presence of cyclic hardening and/or softening phenomenon (Fig. 2 (a) and (b)) during cyclic experiments. In the current study, the structural mild carbon steels S355 exhibits initial cyclic hardening (yield stress, $\sigma_{l}^{0}$, increases to a stable limit, $\sigma_{s}^{0}$ ), followed by cyclic softening (saturated yield stress, $\sigma_{s}$, decreases to the rupture limit, $\sigma_{r}$ ).

The second aspect is the presence of the yield plateau which appears after the first yield in tension as a cause of Luders bands propagation [2] (Fig. 3 (a)). This phenomenon can be idealized as a non-hardening region with the lower bound defined by the yield strain, $\varepsilon y$, and the upper bound defined by the onset of strain hardening, Esh (Fig. 3 (b)). As experimentally observed, the yield plateau disappears when the direction of strain is changed, thus suggesting that the yield plateau is not depending on the plastic strain, $e^{p l}$, but rather on the cumulative plastic strain, $\varepsilon^{-p l}\left(\varepsilon^{-p l}\right.$ is a summation of the plastic strain components during $j$ cycles, $\varepsilon^{-p l}=\sum_{j=1}^{h_{j}} \mathcal{E}^{p l}$; $\varepsilon^{p l}$ is obtained by deducting the elastic strain from the total strain, $\varepsilon^{p l}=\varepsilon-\varepsilon^{l l}$ ).

Considering the above presented phenomena, modelling the cyclic plasticity of steels with yield plateau is difficult due to the complex behaviour. Within this study, the cyclic hardening/softening and the yield plateau phenomena are modelled using a multilinear isotropic hardening model.
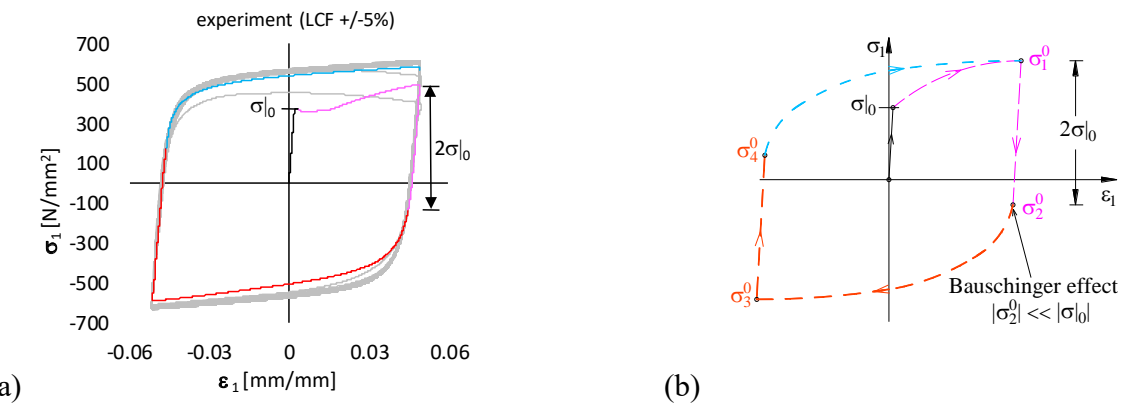

(a)

Fig. 1. B auschinger effect: experiment (a), schematic (b)

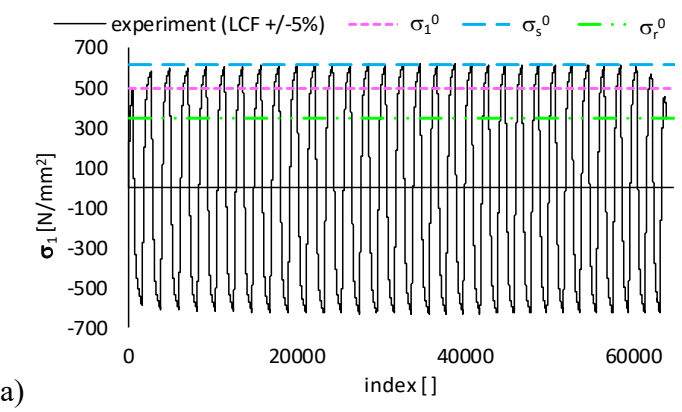

Fig. 2. Cyclic hardening and softening phenomenon: experiment (a), schematic (b) 


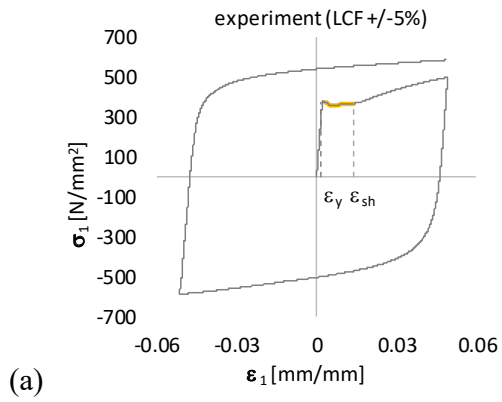

(a)

Fig. 3. Y ield plateau phenomenon: experiment (a), schematic (b)

Both kinematic and isotropic models are implemented in the Finite Element Method (FEM) based software package Abaqus, by using incremental plasticity procedures. In this study, the combined isotropic/kinematic hardening model was chosen to simulate the cyclic behaviour of the mild structural steel S355 based on the conclusions from [3].

\section{Experimental cyclic tests}

Five hourglass-type specimens made of S355 steel grade were machined from a square $30 \times 30 \mathrm{~mm}$ cross-section steel profile. The first specimen, C30-m, was monotonically tested to provide data related to the engineering values of: the yield stress, $\sigma_{y}{ }^{n o m}=366 \mathrm{~N} / \mathrm{mm}^{2}$, the strain at the end of plateau, $\varepsilon s h^{n o m}=1.4 \%$, the ultimate stress, $\sigma_{u}{ }^{n o m}=531 \mathrm{~N} / \mathrm{mm}^{2}$, and its corresponding strain, $\varepsilon u^{n o m}=17.24 \%$. Specimens $C 30-1.4 \%, C 30-3.0 \%$ and $C 30-5.0 \%$ were cyclically strain-controlled tested at constant strain of $1.4 \%, 3.0 \%$ and $5.0 \%$, respectively, while for the last specimen, C30-var, a variable loading protocol was used (two cycles at $\pm 1 \%, \pm 3 \%, \pm 5 \%, \pm 7 \%$, continued by cycles at $\pm 5 \%$ until failure). The experimental cyclic results expressed as engineering stress-strain curves are presented in Fig. 4 (a) to (d).

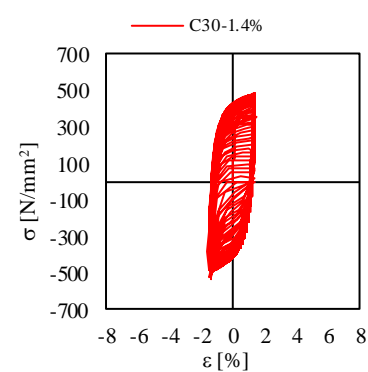

(a)

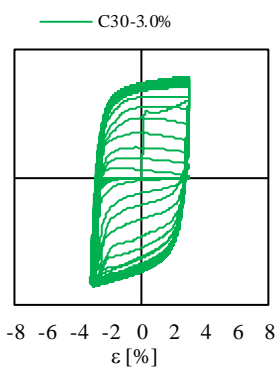

(b)

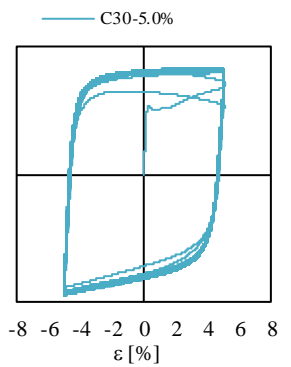

(c)

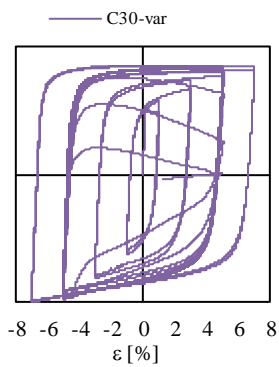

(d)

Fig. 4. Experimental stress-strain curves: C $30-1.4 \%$ (a), C 30-3.0\% (b), C 30-5.0\% (c), C $30-v a r$ (d)

In Fig. 5 the results from the monotonic test $(C 30-m)$ are presented in comparison to the cyclic results (limited cycles) to observe the influence of cyclic loading with respect to strain hardening. In Fig. 6, the results from the three LCF tests $(C 30-1.4 \%, C 30-3.0 \%, C 30-$ $5.0 \%$ ) show that the amount of cyclic hardening differs as a function of strain range, $\Delta \varepsilon$. The amount of cyclic hardening was computed as the difference between the maximum tensile stress value reached during the saturation cycle, $\sigma_{s}^{t}$, and the maximum stress value during the second cycle, $\sigma_{2}^{t}$. The first cycle was not considered for the computation due to the presence of the yield plateau which reduces the amount of kinematic hardening for the first loading phase (in tension). It can be observed that in the case of $C 30-1.4 \%$ and $C 30$ - 
$3.0 \%$ the amount of cyclic hardening is $43 \mathrm{~N} / \mathrm{mm}^{2}$ and it decreases to $29 \mathrm{~N} / \mathrm{mm}^{2}$ in the case of $C 30-5.0 \%$.

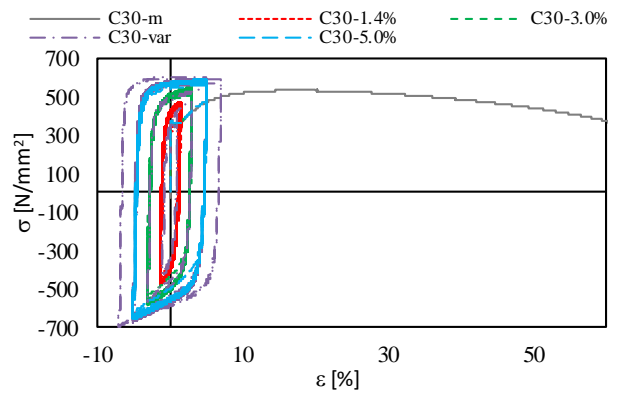

Fig. 5. M onotonic vs. cyclic experimental results

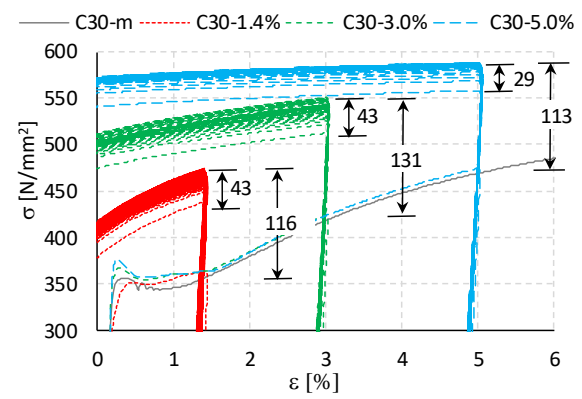

Fig. 6. Strain range dependent cyclic hardening

\section{Material model}

\subsection{Constitutive equations}

The behaviour of the material model used in this paper consists of a linear elastic and a nonlinear plastic component. The elastic behaviour is defined using the isotropic elasticity formulation [1]. Therefore, only the Young's modulus, E, and the Poisson's ratio, $v$, are required to describe the elastic behaviour based on the generalized Hook's law. The "true" (Cauchy) stress tensor, $\sigma$, is defined as a linear function between the fourth-order elasticity tensor, $\mathbf{D}^{\mathrm{el}}$, and the logarithmic strain tensor, $\boldsymbol{\varepsilon}^{\mathrm{el}}, \boldsymbol{\sigma}=\mathbf{D}^{\mathrm{el}} \boldsymbol{\varepsilon}^{\mathrm{el}}$. The plastic behaviour is defined using the pressure-independent plasticity theory [1]. The following constitutive equations and hypotheses were considered to define the plasticity of structural steel:

- Total strain tensor, $\boldsymbol{\varepsilon}$, decomposed into elastic, $\varepsilon^{\mathrm{el}}$, and plastic, $\varepsilon^{\mathrm{pl}}$, parts $\left(\boldsymbol{\varepsilon}=\varepsilon^{\mathrm{el}}+\varepsilon^{\mathrm{pl}}\right)$.

- Yield surface, $F=f(\boldsymbol{\sigma}-\boldsymbol{\alpha})-\sigma^{0}=0$, defined as a function of equivalent stress, $f(\boldsymbol{\sigma}-\boldsymbol{\alpha})$, and yield stress, $\sigma^{0}$ ( $\alpha$ is the backstress tensor). For $F<0$ an elastic stress state is obtained, while $F=0$ yields a plastic stress state; $F>0$ is not admissible.

- von Mises yield criterion, $f(\boldsymbol{\sigma}-\boldsymbol{\alpha})=\left[3 / 2\left(\mathbf{S}-\boldsymbol{\alpha}^{\mathbf{d e v}}\right):\left(\mathbf{S}-\boldsymbol{\alpha}^{\mathbf{d e v}}\right)\right]^{0.5}$, defined as a function of deviatoric stress tensor, $\mathbf{S}$, and deviatoric part of the backstress tensor, $\boldsymbol{\alpha}^{\mathbf{d e v}}$.

- Associated plastic flow rule, $\varepsilon^{\prime p l}=\varepsilon^{\prime}-p l(d F / d \sigma)$, depending on the equivalent plastic strain rate, $\varepsilon^{\prime}-p l=\left[2 / 3\left(\varepsilon^{\prime p l}: \varepsilon^{\prime p l}\right)\right]^{0.5}$.

- Nonlinear isotropic/kinematic hardening rule having the kinematic component defined as a function of nonlinear backstress, $\boldsymbol{\alpha}=\Sigma^{5}{ }_{k=1}\left(\boldsymbol{\alpha}_{\mathbf{k}}\right)$ (where $\boldsymbol{\alpha}_{\mathbf{k}}^{\prime}=C_{k}\left(1 / \sigma^{0}\right)(\boldsymbol{\sigma}-\boldsymbol{\alpha}) \varepsilon^{\prime}-p l-\gamma_{\mathbf{k}} \boldsymbol{\alpha}_{\mathbf{k}} \varepsilon^{\prime}-p l$ ) and the isotropic component, $\sigma^{0}=\sigma \mid 0+\left(\sigma_{i}{ }_{i}-\sigma \mid 0\right)$, defined in a tabular form as a function of equivalent stress defining the yield surface size, $\sigma_{j}$, and equivalent plastic strain, $\varepsilon^{-p l}$.

The combined isotropic/kinematic hardening model is schematically presented in Fig. 7.

Due to the complex nonlinear behaviour of the structural steel under cyclic loading regime, a special attention was given to the hardening rule. The nonlinear kinematic component responsible for the translation of the yield surface in the stress space through the backstress, $\boldsymbol{\alpha}$, is based on the additive decomposed model proposed by Lemaitre and Chaboche (1990) [4]. Five decomposed backstresses, $\boldsymbol{\alpha}_{\mathbf{k}=1.5}$, were considered to properly capture the nonlinear kinematic behaviour, thus allowing for close predictions. Each backstress is characterized by a set of material parameters, $C_{k}$ and $\gamma k$, where $C_{k}$ is the kinematic hardening modulus and $\gamma_{k}$ is the decreasing rate of $C_{k}$ with respect to increasing plastic deformation, $\varepsilon^{\mathrm{pl}}$. For the first backstress, $\boldsymbol{\alpha}_{1}$, (corresponding to small plastic strains) 
large $C_{k}$ and $\gamma_{k}$ values are assigned to assure a smooth and quick transition from the elastic to the plastic behaviour. For the other backstresses, smaller values are assigned to $C_{k}$ and $\gamma_{k}$ since the slope of the stress-strain curve decreases. A linear backstress, $\alpha_{k=5}$ (which corresponds to large plastic strains) is considered for $\gamma_{k=5}=0$.

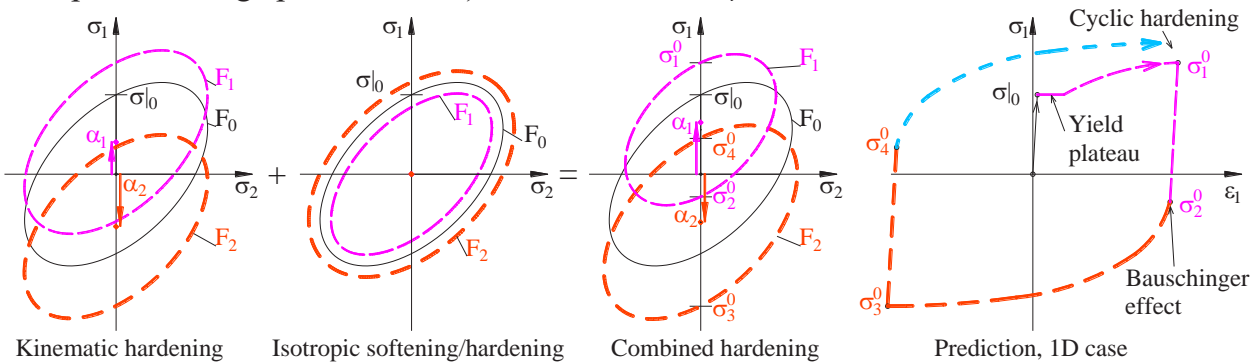

Fig. 7. Combined isotropic/kinematic hardening model

The isotropic hardening component is defined using a multilinear hardening model, which allows for different slopes of the hardening law. Therefore, the yield plateau can be simulated using a negative slope (softening), the cyclic hardening using a positive slope (hardening), while the failure using a negative slope (softening).

\subsection{Calibration procedure}

The calibration procedure refers to the determination of the parameters describing the elastic and plastic behaviour of the material model. The elasticity can be modelled using a Young's modulus of $E=210000 \mathrm{~N} / \mathrm{mm}^{2}$ and a Poisson's ratio of $v=0.3$. As regarding the definition of cyclic plasticity, the parameters describing the kinematic and isotropic hardening laws can be calibrated based on uniaxial cyclic tests under strain control loading regime. The procedure described in [1] was used for calibration and is presented below.

When performing experimental material tests, the measurements are usually expressed as the force $(N)$ per unit undeformed cross-sectional area $\left(A_{0}\right)$ of the specimen, called engineering or nominal stress $\left(\sigma_{\text {nom }}=N / A 0\right)$, and the change in length $(L)$ per unit undeformed monitored length $\left(L_{0}\right)$, called engineering or nominal strain $\left(\varepsilon_{n o m}=L / L\right)$. When defining metal plasticity in Abaqus, true stress, $\sigma=\sigma_{\text {nom }}\left(1+\varepsilon_{\text {nom }}\right)$, and logarithmic strain, $\varepsilon=\ln \left(1+\varepsilon_{\text {nom }}\right)$, relations must be used (Fig. 8 (a)).

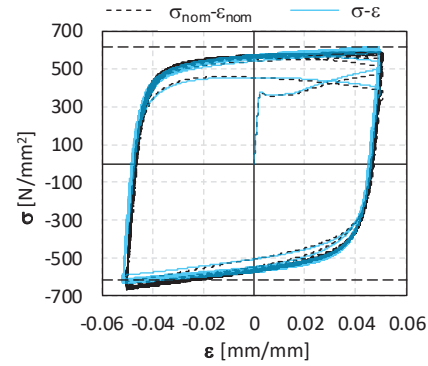

(a)

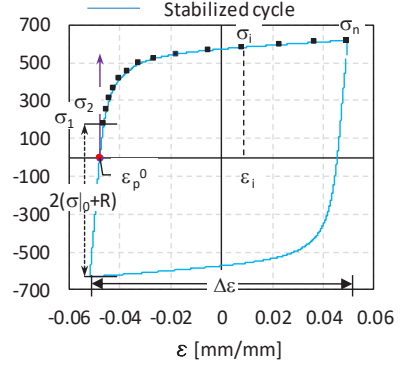

(b)

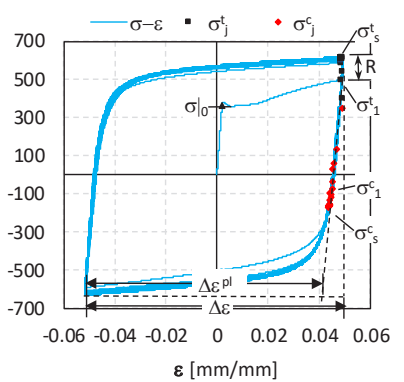

(c)

Fig. 8. Stress-strain curves: nominal vs. true (a), kinematic hardening (b), cyclic hardening (c)

The kinematic hardening component is calibrated by using the "stabilized cycle" procedure defined in [1]. Abaqus automatically determines the material parameters, $C_{k}$.and $\gamma_{k}$, describing the evolution of each of the five backstresses, $\alpha_{k=1.5}$, by using the "built-in" fitting subroutine. Data pairs $\left(\sigma_{i} ; \varepsilon^{p l}\right)$ from the stabilized cycle (corresponding to the 
saturated yield stress, $\sigma_{s}^{0}$ ) must be provided with the strain axis shifted to $\varepsilon_{p}^{0}$ (Fig. $8(\mathrm{~b})$ ). The plastic strain corresponding to $\sigma_{i}$ is expressed as $\varepsilon^{p l}=\varepsilon_{i}-\sigma_{i} / E-\varepsilon_{p}^{0}$, thus $\left(\sigma_{l} ; 0.0\right)$. It is important to properly establish the onset of plastic hardening with respect to the Bauschinger effect: the first data pair $\left(\sigma_{l} ; \varepsilon^{p l}\right)$ corresponds to $2(\sigma / 0+R)$, where $R=\Sigma_{j=3}\left(R_{j}\right)=\Sigma_{j=3}\left(\sigma_{j}^{t}-\sigma_{j-1}^{t}\right)$ is the amount of isotropic hardening recorded until the saturated cycle, $j=1 . . s$. For the first and second cycle $(j=1,2)$ the corresponding amount of cyclic hardening $\left(R_{j}\right)$ is difficult to be determined due to the presence of the yield plateau. Therefore, the values for $R_{j=1,2}$ were determined based on trial and error. The evolution of $R_{j}$ with respect to cycle number is presented in Fig. 9 (a). The calibrated parameters defining the kinematic hardening component are presented in Table 1. The evolution of the backstress $(\alpha)$ with respect to plastic strain $\left(\varepsilon^{p l}\right)$ is graphically presented in Fig. $9(\mathrm{~b})$.

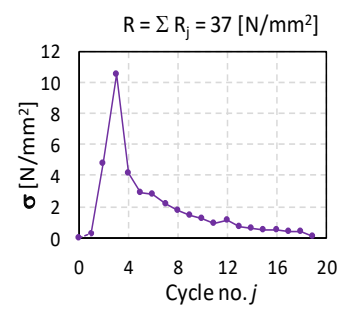

(a)

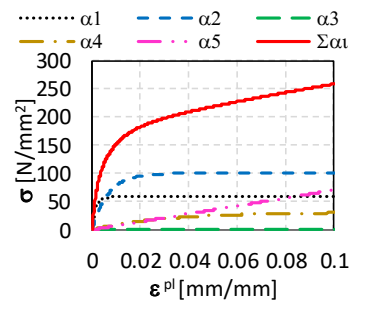

(b)

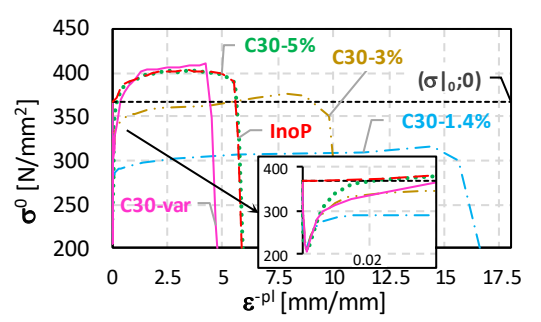

(c)

Fig. 9. Evolution of $R_{i}(\mathrm{a})$; evolution of $\alpha(\mathrm{b})$; evolution of $\sigma^{0}$ (c)

Table 1. Calibrated parameters defining kinematic hardening component $\left(\sigma \mid 0\right.$ and $C_{k}$ in $\left.N / \mathrm{mm}^{2}\right)$.

\begin{tabular}{|c|c|c|c|c|c|c|c|c|c|c|}
\hline$\sigma \mid \boldsymbol{\theta}$ & $\mathbf{C}_{1}$ & $\gamma_{\mathbf{1}}$ & $\mathbf{C}_{2}$ & $\gamma_{\mathbf{2}}$ & $\mathbf{C}_{3}$ & $\gamma_{3}$ & $\mathbf{C}_{4}$ & $\gamma_{4}$ & $\mathbf{C}_{\mathbf{5}}$ & $\gamma_{\mathbf{5}}$ \\
\hline 367.08 & 41513 & 697 & 15152 & 137.5 & 600 & 4.6 & 255 & 2.2 & 195 & 0 \\
\hline
\end{tabular}

The isotropic hardening component is calibrated using the entire data from the symmetric strain-controlled experiment. Thus, the evolution of the size of the yield surface $\left(\sigma^{0}\right)$ over the entire loading history can be determined. For the current cycle $(j>1)$, the strain range is $\Delta \varepsilon$ (Fig. $8(\mathrm{c})$ ), and the plastic strain range can be obtained by deducting the elastic part, $\Delta \varepsilon^{p l}=\Delta \varepsilon-2 \sigma_{j}^{+} / E$. Special consideration needs to be taken in the case of the first (tensile) cycle $(j=1)$, since the elastic strain range is $\Delta \varepsilon_{1}=\Delta \varepsilon / 2$ and the plastic strain range is $\varepsilon l^{p l}=\Delta \varepsilon_{l}-\sigma^{t} l / E$. In Abaqus, the size of the yield surface at corresponding equivalent plastic strains must be defined by providing data pairs $\left(\sigma_{j}^{0} ; \varepsilon^{-p l}\right)$. The equivalent stress is obtained from the peak tensile stress $\left(\sigma_{j}^{t}\right)$ by eliminating the kinematic component, $\sigma_{j}=\sigma_{j}^{t}-\alpha_{j}$, where $\alpha_{j}=\left(\sigma_{j}^{t}+\sigma_{j}^{c}\right) / 2$, with $\sigma_{j}^{c}$ the compressive yield stress corresponding to cycle $j$. The corresponding equivalent plastic strain $\left(\varepsilon^{-p l}\right)$ can be determined as a function of cycle number and plastic strain range, $\varepsilon^{-p l}{ }_{j}=0.5(4 j-3) \Delta \varepsilon^{p l}$. For the first cycle, $\varepsilon^{-p l}{ }_{l}=\varepsilon^{p l}{ }_{l}$.

Due to the limitations of the isotropic model [1], the calibration needed to be performed for each cyclic experiment, thus resulting different input curves $\left(\sigma_{j}^{0} ; \varepsilon^{-p l}\right)$, as presented in Fig. 9 (c) (C30-1.4\%, C30-3.0\%, C30-5.0\%, C30-var). It can be observed that the isotropic input is loading history dependent. In addition, two isotropic inputs were also obtained: $(\sigma / 0 ; 0.0)$ - no isotropic hardening; InoP - isotropic hardening without initial yield plateau.

\section{Application in a finite element model}

One cubic finite element of type C3D8I (incompatible mode eight-node linear brick element) was used as FEM model for the material calibration process (Fig. 10 (a)). The 
reason behind this choice is related to the strain ranges of the loading protocols which are smaller than the value of the strain corresponding to the ultimate stress $\left(\varepsilon^{u}\right.$ nom $\left.=17.24 \%\right)$, and therefore no necking appears. Thus, material failure mode is caused by low-cycle fatigue.

No transversal deformations were limited $\left(U_{x}=U_{z}=\right.$ free, where $U_{i}$ is translational degree of freedom corresponding to axis i) to allow for the Poisson effect. The loading protocol is applied at the top $\left(U_{y}\right)$ as displacement control. By using this procedure, the required strain amplitudes can be easily simulated. Different combinations of kinematic (KIN) and isotropic (ISO) hardening were used to prove the necessity of using a multilinear isotropic hardening capable of simulating both the yield plateau and the cyclic hardening, as presented in Fig. 10 (b) to (d). The Static-General Abaqus procedure with nonlinear effects of large deformations and displacements was used to run the cyclic analyses.

The predictions using the complex combined hardening model are presented in Fig. 11. As a general observation, the material model can reproduce the experimental results with a high level of accuracy, including bot cyclic behaviour and failure mode of the specimens.

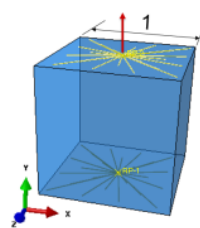

C3D8I

$\mathrm{Ux}=\mathrm{Uz}=$ free, $\mathrm{Uy}=$ load control

(a)

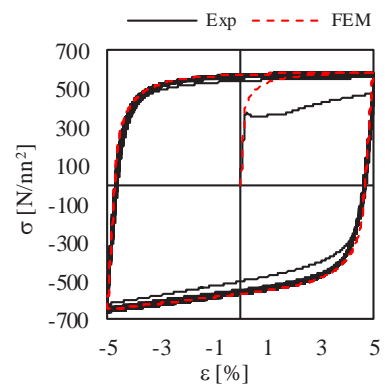

$\mathrm{KIN}=$ stabilized ISO $=$ no

(b)

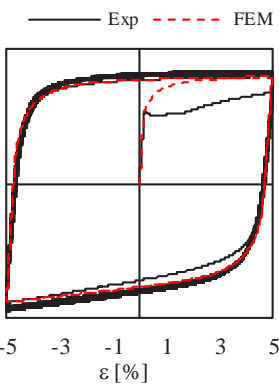

$\mathrm{KIN}=$ stabilized ISO $=(\sigma \mid 0 ; 0.0)$

(c)

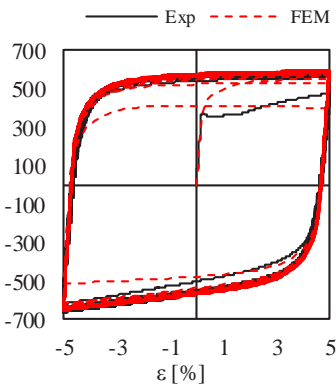

$\mathrm{KIN}=$ stabilized ISO $=$ InoP (no-plateau)

(d)

Fig. 10. FEM model (a); unsatisfactory predictions using inappropriate isotropic input (b), (c), (d)

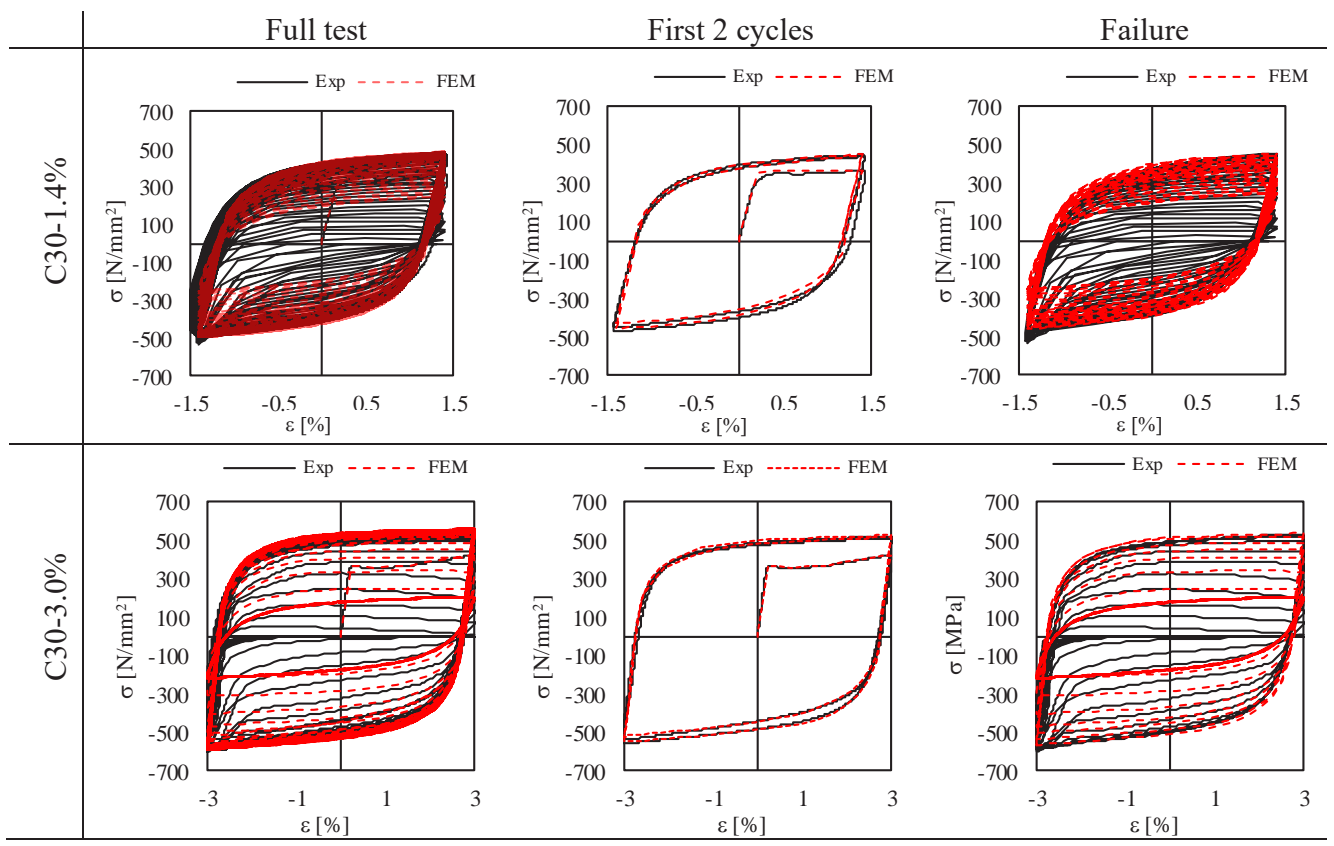




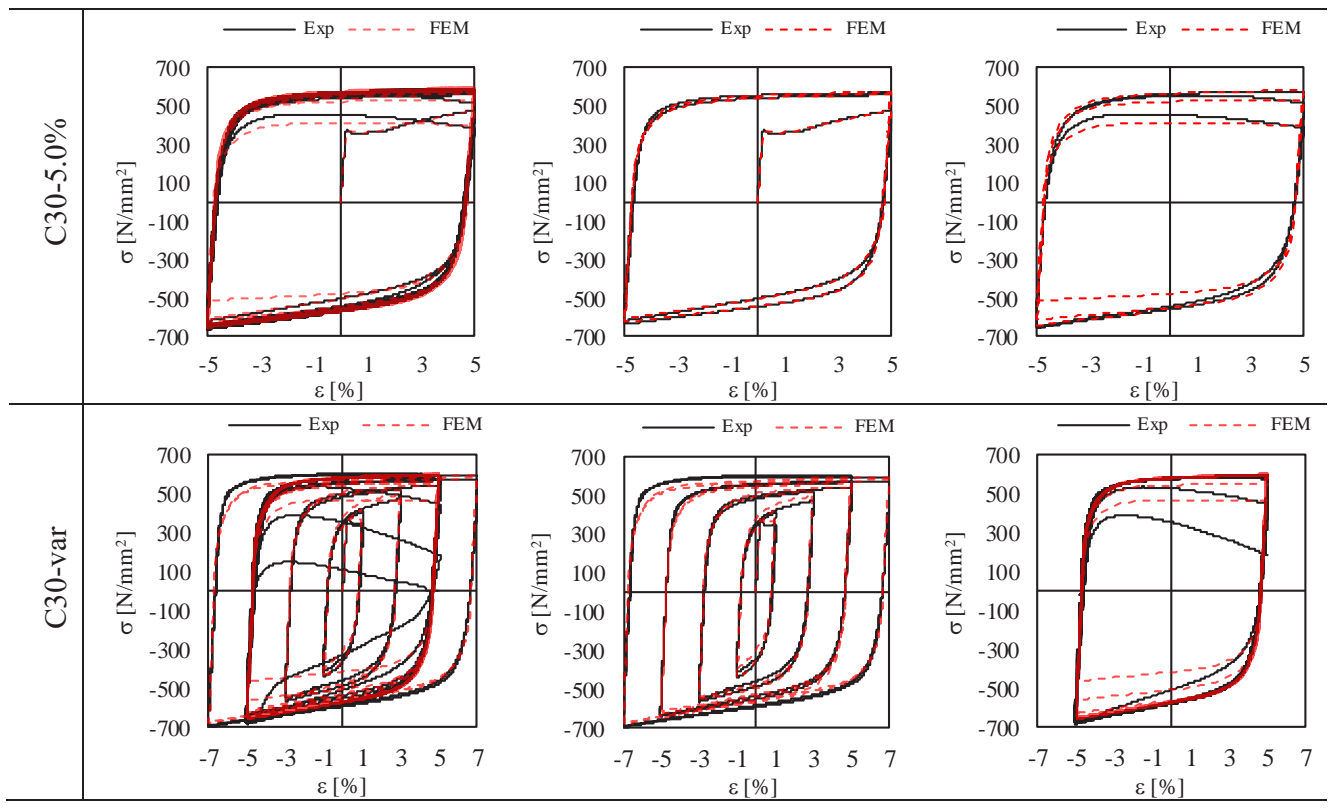

Fig. 11. FEM predictions using the appropriate definition of the combined hardening model

\section{Conclusions}

The proposed modelling technique of the cyclic response of structural steel for FEM analyses using the Abaqus "built-in" material models proved to assure reliable results. The material model can properly simulate the main features of mild carbon steel S355.

The calibration of the elastic and plastic behaviour is based on experimental results. Five coupons were tested: one uniaxial monotonic test and four uniaxial cyclic tests (three tests under symmetric strain control and one under variable strain control). The dependency of the cyclic hardening with respect to the strain range was experimentally observed.

The combined isotropic/kinematic hardening model is used to model metal plasticity under cyclic loading. The kinematic hardening definition is consistent for all loading cases. The isotropic hardening definition is not consistent for all loading cases, therefore is loading history dependent. The calibrated FEM results closely predict the experimental results, with respect to the cyclic behaviour and the failure mode.

Further studies aim at extending the experimental database through numerical testing, by varying the material parameters.

The research leading to these results has received founding from the MEN-UEFISCDI grant Partnerships in priority areas PN II, contract no. 99/2004 IMSER: "Implementation into Romanian seismic resistant design practice of buckling restrained braces."

\section{References}

1. ABAQUS “ABAQUS Documentation”, Dassault Systèmes, Providence, RI, USA, (2014).

2. E.O. Hall. Y ield point phenomena in metals and alloys. New Y ork: Plenum Press (1970).

3. C.I. Zub, A. Stratan, A. Dogariu, D. Dubina. Development of a finite element model for a buckling restrained brace, Procc. of the Romanian A cademy, series A, no. 19(4) (2018).

4. J. Lemaitre, J.L. Chaboche, M echanics of Solid M aterials, Cambridge U niversity Press (1990). 